\title{
O impacto das fraturas do quadril no SUS 2008 - 2017: O papel do ortopedista
}

\section{The Impact of Hip Fractures in the Public Health System in Brazil (SUS) 2008 - 2017: The Orthopedist Task}

\author{
Bernardo Stolnicki ${ }^{10}$ Bruno Casaes Teixeira ${ }^{2,3}$ \\ ${ }^{1}$ Setor de Doenças Osteometabólicas, Departamento de Ortopedia, \\ Hospital Federal de Ipanema, Rio de Janeiro, RJ, Brasil \\ ${ }^{2}$ Amgen Biotecnologia do Brasil Ltda, São Paulo, SP, Brasil \\ 3 Faculdade de Saúde Pública, Universidade de São Paulo, São Paulo, \\ Brasil
}

\begin{abstract}
Endereço para correspondência Bernardo Stolnicki, MD, Setor de Doenças Osteometabólicas, Departamento de Ortopedia, Hospital Federal de Ipanema, Rua Barão da Torre, 350/201, Ipanema, Rio de Janeiro, RJ, 22411-000, Brasil (e-mail: stolnick@hotmail.com).
\end{abstract}

Rev Bras Ortop 2022;57(4):552-559.

\section{Resumo \\ Palavras-chave \\ - fraturas do quadril \\ - reabilitação \\ - Datasus \\ - prevenção secundária}

Objetivo O presente estudo tem como objetivo descrever o perfil de hospitalização e reabilitação ambulatorial de pacientes com idade $\geq 50$ anos por fratura de quadril no Sistema Público de Saúde no Brasil (SUS).

Métodos Trata-se de um estudo transversal de pacientes internados por fratura de quadril no SUS entre 2008 e 2017. Os dados incluíram 441.787 internações relacionadas à fratura de quadril do banco de dados de internação (SIH/DATASUS) e dados de pacientes submetidos à reabilitação do banco de dados ambulatorial (SIA/DATASUS). Resultados A maioria das hospitalizações relacionadas à fratura de quadril (83,5\%) ocorre em pessoas $\geq 50$ anos, com um crescimento médio anual de 5,6\% nas hospitalizações relacionadas à fratura de quadril (HRFQ). Os custos para o governo cresceram na mesma proporção e atingiram quase 130 milhões de reais em 2017, embora com uma redução de $13,6 \%$ no custo médio por hospitalização. Além do impacto financeiro, as fraturas de quadril resultam em uma taxa de mortalidade hospitalar em torno de $5,0 \%$ em pacientes $\geq 50$ anos. Além disso, o percentual de pacientes submetidos à reabilitação relacionada à fratura de quadril aumentou de 2008 (14,0\%) para 2012 (40,0\%) e permaneceu estável após esse período.

Conclusões $\mathrm{O}$ aumento progressivo da incidência de fraturas de quadril mostra o impacto financeiro e social e a necessidade de ações imediatas para evitar essa tendência crescente. As fraturas de quadril são um risco para fraturas secundárias, a prevenção é crucial e o ortopedista desempenha um papel central nesse processo.
Estudo desenvolvido no Setor de Doenças Osteometabólicas, Departamento de Ortopedia, Hospital Federal de Ipanema, Rio de Janeiro, RJ, Brasil. recebido

14 de Outubro de 2019

aceito

15 de Abril de 2020

Publicado on-line

Setembro 30, 2020
DOI https://doi.org/ 10.1055/s-0040-1713762. ISSN 0102-3616.
(C) 2020. Sociedade Brasileira de Ortopedia e Traumatologia. All rights reserved.

This is an open access article published by Thieme under the terms of the Creative Commons Attribution-NonDerivative-NonCommercial-License, permitting copying and reproduction so long as the original work is given appropriate credit. Contents may not be used for commercial purposes, or adapted, remixed, transformed or built upon. (https://creativecommons.org/ licenses/by-nc-nd/4.0/)

Thieme Revinter Publicações Ltda., Rua do Matoso 170, Rio de Janeiro, RJ, CEP 20270-135, Brazil 


\begin{abstract}

\section{Keywords}

- hip fractures

- rehabilitation

- datasus

- secondary prevention

Objective The present study intends to describe the profile of hospitalization and ambulatory rehabilitation of patients $\geq 50$ years old due to hip fracture in the Brazilian Public Health System (SUS, in the Portuguese acronym).

Methods This is a cross-sectional study of patients hospitalized due to hip fracture in the SUS between 2008 and 2017. Data included 441,787 hip fracture-related hospitalizations from the hospitalization database of the department of informatics of the Brazilian Unified Health System (SIH/DATASUS, in the Portuguese acronym), and data of patients who underwent rehabilitation from the ambulatory database of the department of informatics of the Brazilian Unified Health System (SIA/DATASUS, in the Portuguese acronym.).

Results Most of hip fracture-related hospitalizations (83.5\%) happen to people $\geq$ 50 years old, with an average annual growth of $5.6 \%$ in hip fracture-related hospitalizations. The costs for the government have been growing in the same proportion and reached almost BRL 130 million in 2017, although with a 13.6\% decrease in average cost per hospitalization. Besides the financial impact, hip fractures result in an inhospital mortality rate around $5.0 \%$ in patients aged $\geq 50$ years old. In addition, the percentage of patients that have undergone hip fracture-related rehabilitation increased from 2008 (14.0\%) to 2012 (40.0\%), and remained stable after that.

Conclusions The progressive increase in the incidence of hip fractures shows the financial and social impact, and the need for immediate actions to prevent this rising trend. Hip fractures are a risk for secondary fractures, the prevention is crucial, and the orthopedist plays a central role in this process.
\end{abstract}

\section{Introdução}

As fraturas osteoporóticas são uma crescente condição de saúde pública, com uma incidência crescente à medida que a população envelhece. As fraturas de quadril estão fortemente associadas a morbimortalidade substancial, baixa densidade mineral óssea, implicam em custos mais altos para reparo e causam mais incapacidade do que outras fraturas. ${ }^{1,2}$ Apesar de todas as fraturas osteoporóticas estarem associadas ao aumento do risco de mortalidade por 5 anos, a fratura de quadril tem o pior prognóstico. A mortalidade associada à fratura de quadril permanece elevada por até 10 anos, ${ }^{3}$ e são previstas fraturas subsequentes, especialmente durante o $1^{\circ}$ ano. ${ }^{4} \mathrm{O}$ risco de fraturas após uma fratura de quadril que surge com um trauma mínimo é quase quatro vezes maior. ${ }^{5}$ Um estudo anterior desenvolvido pelo nosso grupo mostrou que $86 \%$ dos pacientes com fratura proximal do quadril tinham pelo menos uma fratura anterior. ${ }^{6}$ Estudos epidemiológicos demonstraram que as fraturas proximais do fêmur levam a 20\% de mortalidade em 12 meses, incapacidade funcional permanente em $30 \%$ e incapacidade de caminhar em $40 \%$ dos indivíduos. ${ }^{2}$

Medidas preventivas, como iniciar o tratamento logo após uma fratura no quadril, podem diminuir a recorrência de fraturas em torno de 30 a $60 \%{ }^{7}$ e reduzir os fatores de risco de mortalidade, como idade, uso de medicamentos, mau equilíbrio e condições crônicas, que desempenham papel crítico na recorrência de fraturas de quadril, especialmente para aqueles pacientes que precisam de reabilitação após fraturas anteriores. $^{8}$ Vários fatores afetam a recuperação após uma fratura de quadril, mas há evidências substanciais de que os exercícios são benéficos e mostram resultados promissores da reabilitação. ${ }^{9}$ Estudos sistemáticos de revisão e metanálise demonstraram os benefícios da reabilitação e a recomendam após a ocorrência de fraturas de quadril. ${ }^{10}$

Desde 1994, vários estudos brasileiros descrevem a epidemiologia das fraturas osteoporóticas no Brasil. ${ }^{11}$ A maioria dos estudos sobre fratura de quadril relata incidência e mortalidade regionalmente e por um período limitado de tempo. ${ }^{12-15}$ A alta incidência de fraturas de quadril - que, por sua vez, leva a custos elevados para o governo brasileiro - deve ser uma prioridade da saúde pública. ${ }^{14,16}$ Além disso, a incapacidade dos pacientes retornarem à comunidade é um componente adicional dos custos associados à fratura de quadril, ${ }^{17}$ que destaca ainda mais a importância da reabilitação.

Faltam dados nacionais amplos sobre a incidência das fraturas de quadril no Brasil. Além disso, a proporção de pacientes submetidos a reabilitação após hospitalização por fratura de quadril é desconhecida. Portanto, o presente estudo tem como objetivo descrever o perfil de internação por fratura de quadril e reabilitação ambulatorial de pacientes $\geq 50$ anos no Sistema Público de Saúde no Brasil (SUS).

\section{Métodos}

\section{Desenho do Estudo e Fonte de Dados}

Trata-se de um estudo transversal de pacientes adultos internados por fratura de quadril no SUS. Os dados foram extraídos primeiro dos registros do Sistema de Informações Hospitalares (SIH/DATASUS). Além dos dados de internação, foram extraídos dados do Sistema de Informações Ambulatoriais (SIA/DATASUS) 
referentes a todos os procedimentos ambulatoriais individualizados relacionados a um paciente submetido a reabilitação após fratura de quadril. Os SIH/DATASUS e SIA/DATASUS são bancos de dados que cobrem $\sim 160$ milhões de pessoas no SUS, com informações de todas as internações e procedimentos ambulatoriais reembolsados pelo Governo Federal, e têm como objetivo fornecer controle financeiro para fins de auditoria. O sistema inclui a Classificação Internacional de Doenças (CID-10) e dados demográficos, entre outras informações.

\section{População do Estudo}

Os critérios de inclusão dos pacientes no banco de dados de internação (SIH/DATASUS) foram pacientes hospitalizados que sofreram fratura de fêmur de janeiro de 2008 a dezembro de 2017, com um dos seguintes códigos da CID-10 como causa de hospitalização (campo DIAG_PRINC): S72.0 (fratura da cabeça e pescoço do fêmur), S72.1 (fratura pertrocantérica) ou S72.2 (fratura subtrocantérica do fêmur). Em seguida, foram coletados dados do banco de dados ambulatorial (SIA/DATASUS) para pacientes submetidos à reabilitação (código de procedimento 0302050019 - Atendimento fisioterapêutico para pacientes pré e pós-cirúrgicos com distúrbios osteomusculares) relacionados a uma fratura de quadril (CID 10: S72.0, S72.1 e S72.2). A - Fig. 1 descreve o fluxograma dos critérios de elegibilidade.

\section{Análise de Dados}

As tabelas do SIA têm identificação anônima que permite a contagem direta de pacientes. No entanto, para o banco de dados SIH, não há campo de identificação e o número de pacientes que foram incluídos apenas uma vez na folha de dados foi estimado a partir do bloco criado pelos campos sexo, data de nascimento e CEP. Avaliamos a frequência de hospitalizações relacionadas à fratura de quadril (HRFQ) na população por ano e de acordo com idade e sexo. Também foram observados o percentual de hospitalizações que terminaram com óbito, duração da internação e procedimentos relacionados à fratura de quadril. Posteriormente, foram avaliadas as porcentagens de pacientes submetidos a procedimentos de reabilitação ambulatorial. Os dados descritivos foram relatados como média \pm desvio padrão (DP) para variáveis e frequências contínuas e porcentagens para variáveis categóricas.

\section{Resultados}

Dados de 2.046 hospitais brasileiros, incluindo 441.787 hospitalizações relacionadas à fratura de quadril (HRFQ) ocorridos entre 2008 e 2017, foram avaliados. A idade média dos pacientes no momento da internação foi de $68.5 \pm 20.9$ anos. A - Tabela 1 descreve as características da população estudada.

A - Fig. 2 mostra HRFQ por ano naqueles $<50$ anos e $\geq 50$ anos. A HRFQ em pessoas $<50$ anos tem sido estável, enquanto a maior parte do crescimento é atribuída a pessoas $\geq 50$ anos. Houve um crescimento médio anual de $5,6 \%$ na HRFQ em pessoas $\geq 50$ anos, com menor crescimento de 2009 a 2010 e crescimento máximo de 2015 a 2016 (12,2\%).

A distribuição das fraturas por gênero varia de acordo com a idade. Mais fraturas de quadril foram observadas em homens entre 50 e 60 anos. Uma prevalência semelhante

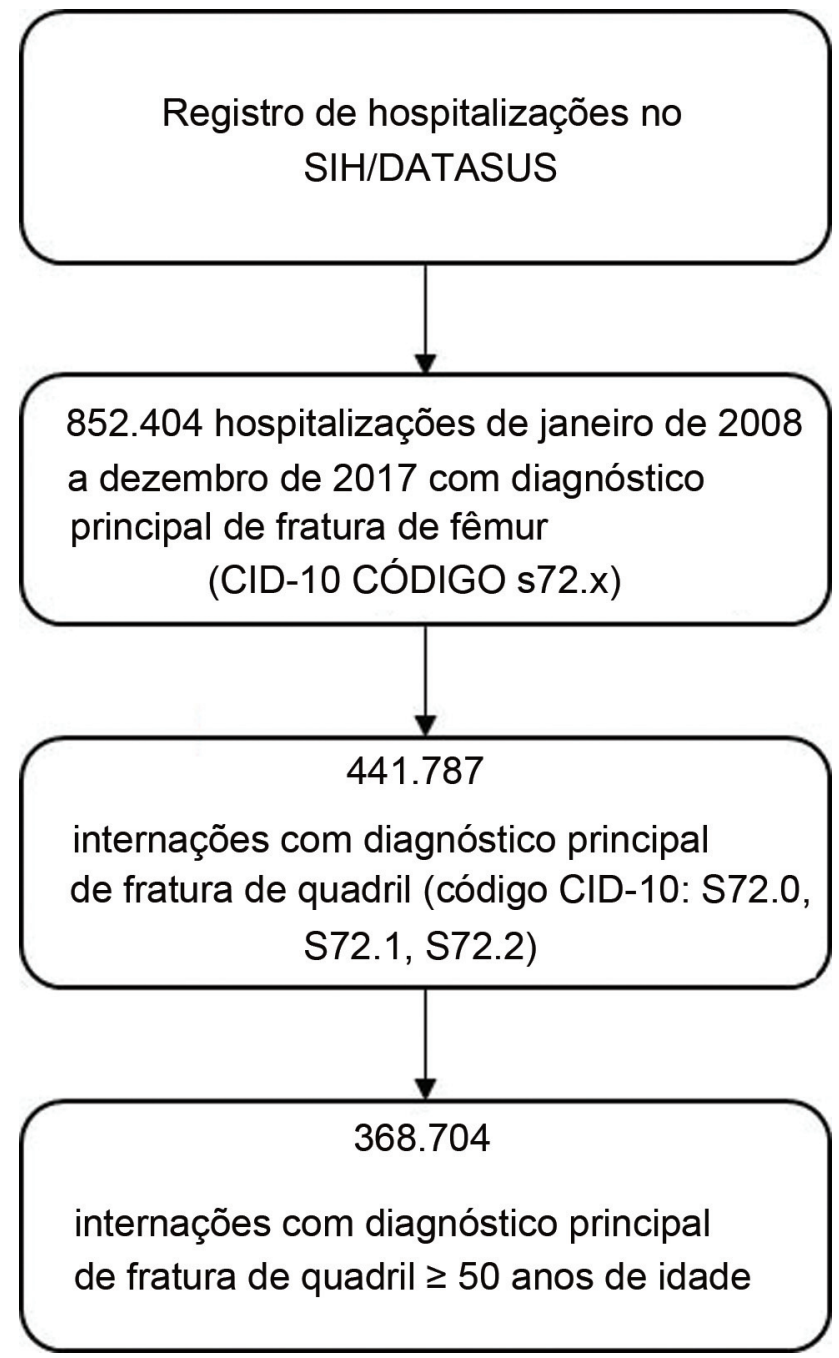

Fig. 1 Fluxograma da elegibilidade da população do estudo.

Tabela 1 Características da População Estudada $(n=441.787)$

\begin{tabular}{|l|l|l|}
\hline Variáveis & Frequência & Percentatual \\
\hline $\begin{array}{l}\text { Pacientes } \geq 50 \text { anos no } \\
\text { momento da internação }\end{array}$ & 368.704 & $83,5 \%$ \\
\hline Gênero & & \\
\hline Masculino & 184.667 & $41,8 \%$ \\
\hline Feminino & 257.120 & $58,2 \%$ \\
\hline Ano de internação & & \\
\hline $2008-2009$ & 74.592 & $16,9 \%$ \\
\hline $2010-2011$ & 78.920 & $17,9 \%$ \\
\hline $2012-2013$ & 85.013 & $19,2 \%$ \\
\hline $2014-2015$ & 93.848 & $21,2 \%$ \\
\hline $2016-2017$ & 109.414 & $24,8 \%$ \\
\hline
\end{tabular}

entre os sexos é observada entre 60 e 65 anos de idade, mas é maior em mulheres $\geq 65$ anos (-Fig. 3).

De 2008 a 2017, a proporção de HFRQ que resultou em morte durante o período de hospitalização em pacientes $\geq 50$ 


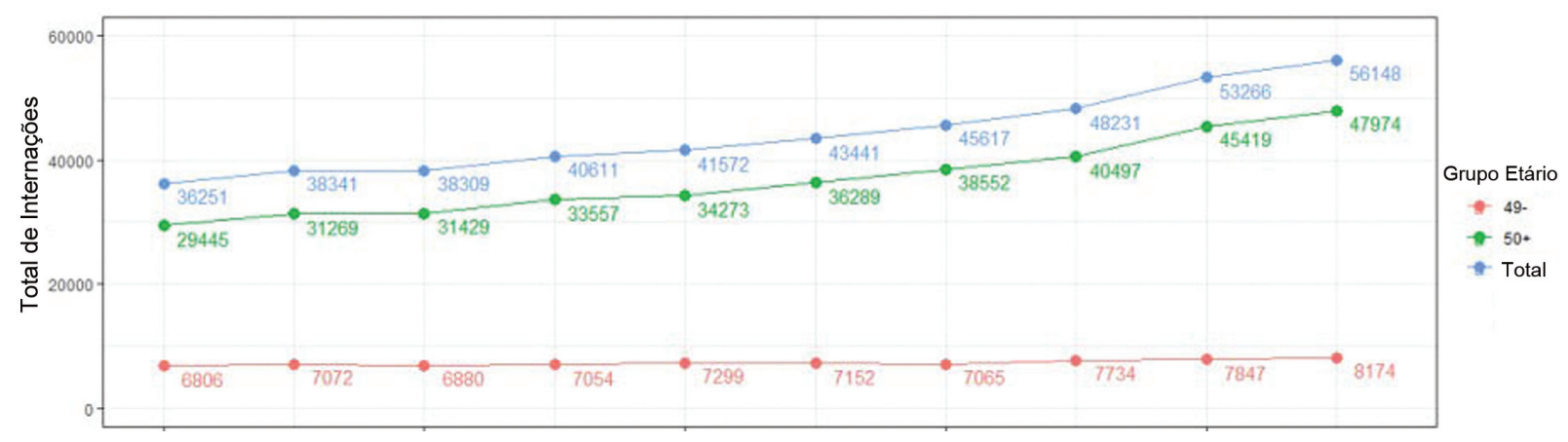

Fig. 2 Internações por Fratura de Quadril por ano. Brasil 2008-2017.
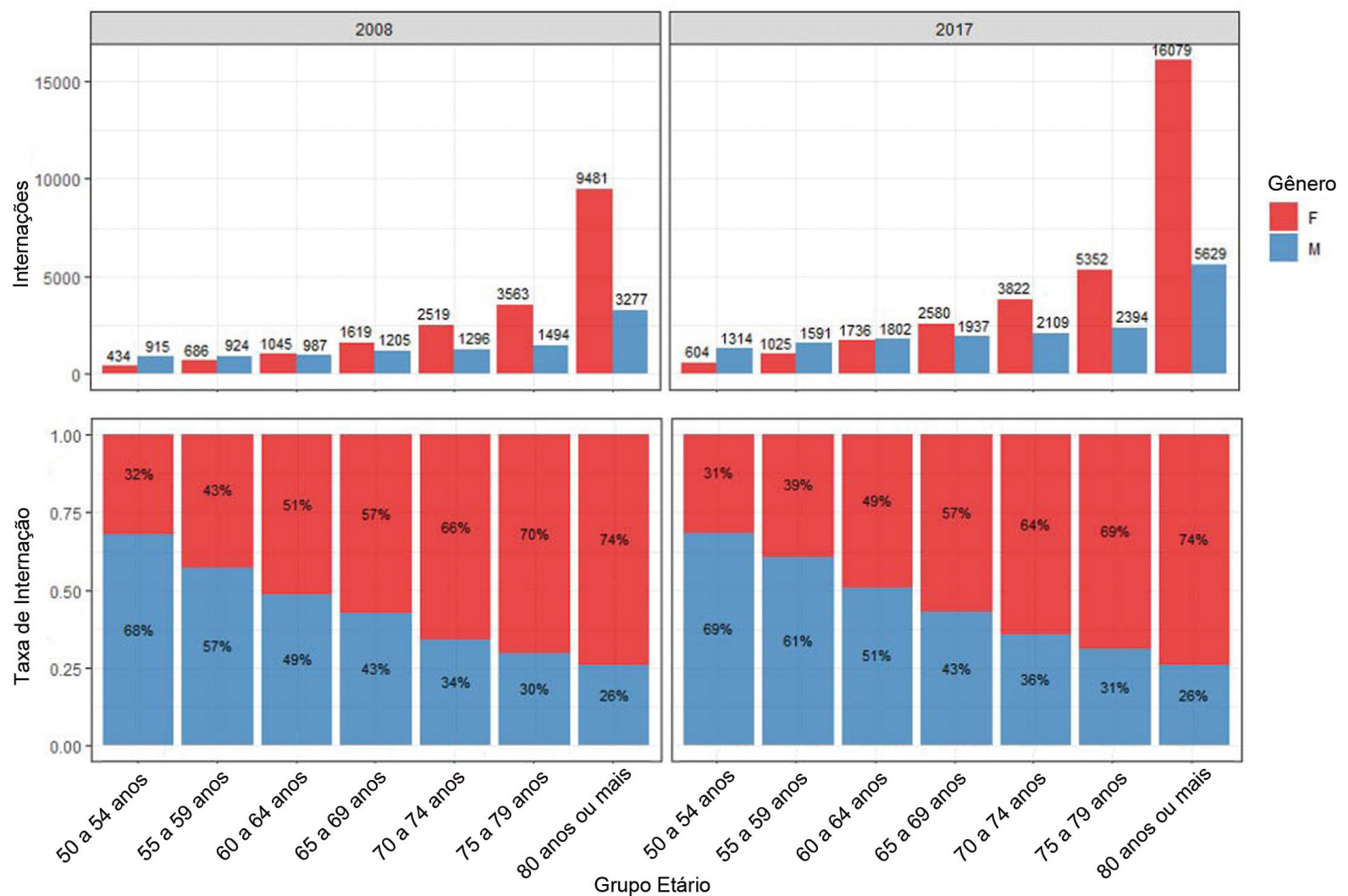

Fig. 3 Distribuição etária por gênero das internações por fratura de quadril por ano. Brasil 2008-2017.

anos de idade ficou entre 4,0 e 5,0\% (-Fig. 4). No entanto, quando esses resultados são avaliados considerando sexo e faixa etária, o perfil das taxas de mortalidade mudou de 2008 para 2017. Geralmente, a taxa de mortalidade foi maior nos homens na maioria das faixas etárias. As taxas de mortalidade em homens foram consideravelmente mais altas para aqueles $\geq 65$ anos, atingindo 8,6\% em homens > 80 anos, em comparação com 6,9\% em mulheres na mesma faixa etária. (-Fig.5).

É importante observar que o tempo de internação é maior nos pacientes que morreram durante a internação e, consequentemente, os custos da internação também são maiores em comparação com os que recebem alta hospitalar. Apesar do tratamento e do desenvolvimento de tecnologia nos últimos 10 anos, o tempo de internação não mudou de 2008 para 2017, nem para os pacientes que receberam alta do hospital nem para os que morreram (-Tabela 2 ).
A - Tabela 3 descreve os 10 principais procedimentos individuais realizados para HRFQ durante a hospitalização, de acordo com os custos nos anos de 2008 e 2017. Os procedimentos de tratamento relacionados às maiores taxas de mortalidade em pacientes $\geq 50$ anos foram os conservadores, tanto em 2008 quanto em 2017. Observamos que o procedimento individual imobilização, nos membros inferiores, um tratamento conservador de fraturas listado como sétimo no ranking em 2008, e com maior taxa de mortalidade, não está listado em 2017, o que indica uma mudança no manejo das fraturas em membros inferiores.

O número estimado de pacientes que foram incluídos apenas uma vez na folha de dados e passaram por uma HRFQ aumentou continuamente desde 2008. Além disso, o percentual de pacientes que passou por uma reabilitação relacionada à fratura de quadril aumentou de 2008 (14,0\%) para 2012 (40,0\%). No 


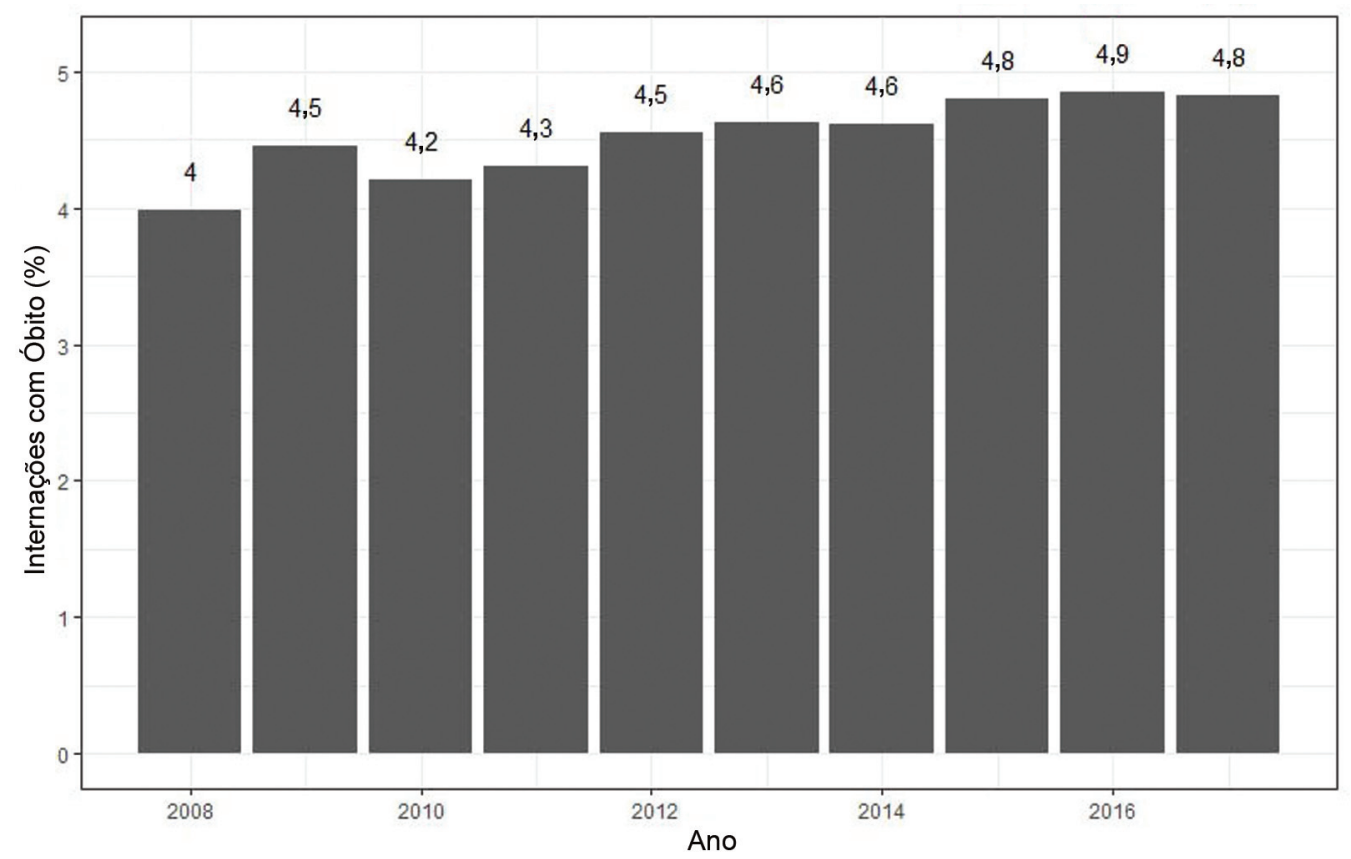

Fig. 4 Percentual de hospitalizações que terminaram em óbito por ano ( $\geq 50$ anos). Brasil 2008 - 2017.

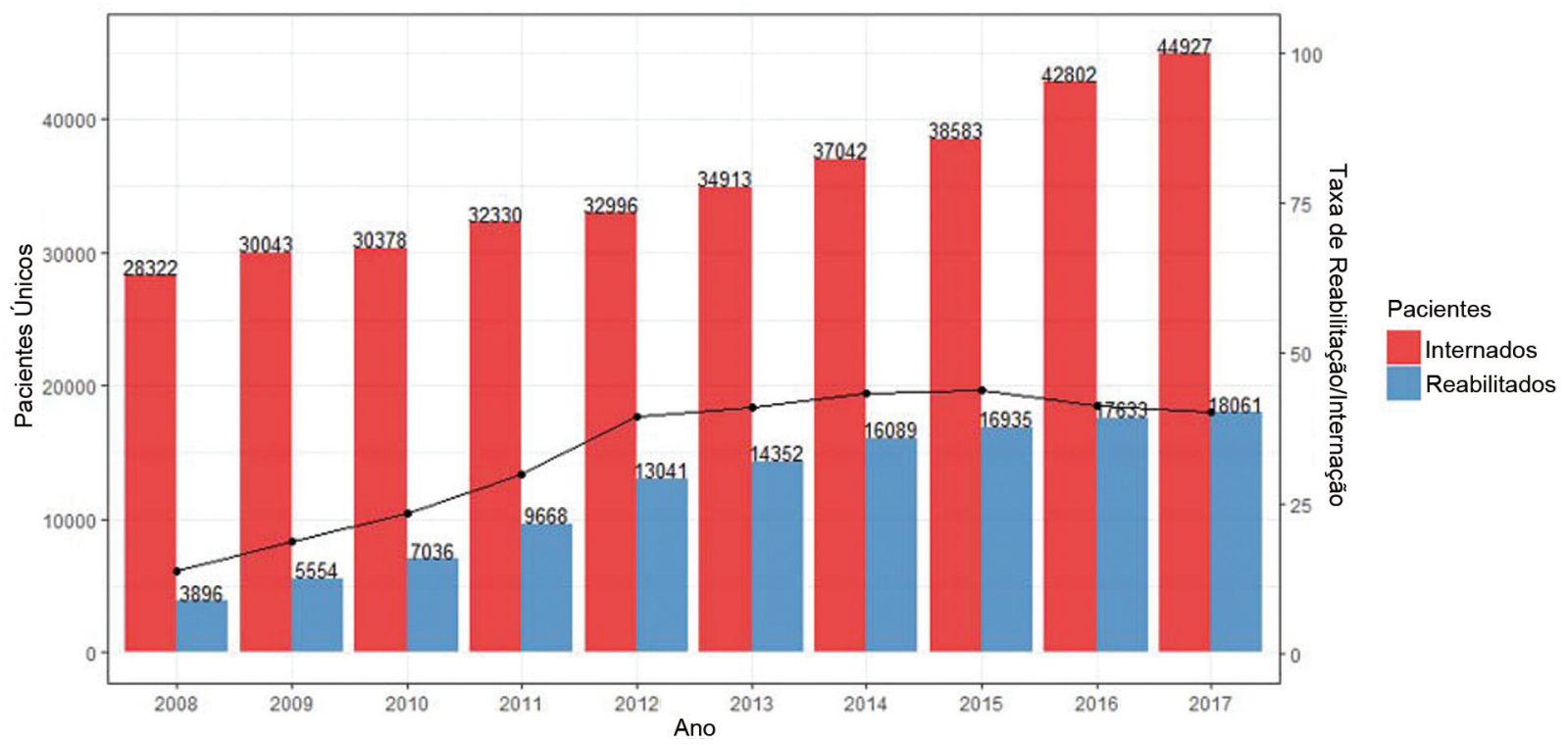

Fig. 5 Contagem de pacientes na hospitalização relacionada à fratura do quadril e na reabilitação relacionada à fratura do quadril ( $\geq 50$ anos). Brasil $2008-2017$.

Tabela 2 Duração e custos da hospitalização de acordo com o resultado da hospitalização em pacientes $\geq 50$ anos - Brasil 2008 2017

\begin{tabular}{|l|l|l|l|}
\hline & & Alta do hospital & Óbitos \\
\hline 2008 & $\mathrm{n}$ & 28.272 & 1.173 \\
\hline & Tempo médio de internação & $8,0 \pm 7,5$ & $11,0 \pm 13,9$ \\
\hline & Custo médio (BRL) & $4.433 \pm 5.006$ \\
\hline 2017 & $\mathrm{n}$ & $3.016 \pm 1.798$ & 2.316 \\
\hline & Tempo médio de internação & 45.658 & $10,5 \pm 11,8$ \\
\hline & Custo médio (BRL) & $8,2 \pm 7,9$ & $4.090 \pm 4.425$ \\
\hline
\end{tabular}

*Valor em Reais brasileiros. Valores corrigidos pela inflação com o fator 1.608 de acordo com o IGP-M. 


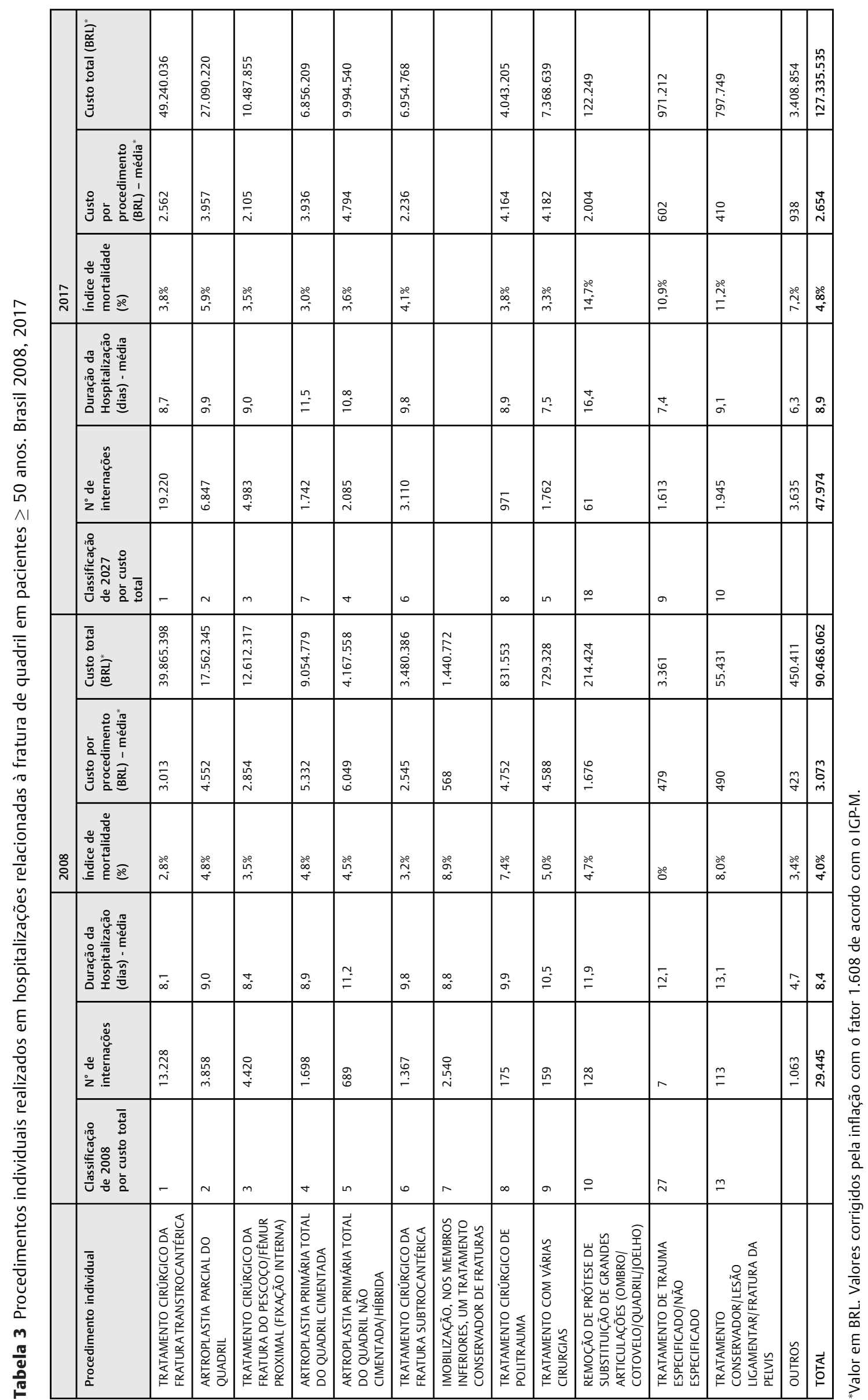


entanto, a partir de 2012, o percentual de pacientes que tiveram reabilitação relacionada à fratura de quadril permaneceu estável até 2017. É importante notar que, apesar desse aumento, atualmente, menos da metade dos pacientes hospitalizados por fratura de quadril irá para um centro de reabilitação.

\section{Discussão}

O presente estudo avaliou dados do SUS de 2008 a 2017. De acordo com nossos resultados, houve uma média de 5,6\% de crescimento anual nas fraturas de quadril atribuídas a pessoas $\geq$ 50 anos. Considerando que o Brasil teve um crescimento anual da população de $0,9 \%$ no mesmo período, ${ }^{18}$ o aumento na ocorrência de fraturas de quadril é maior que o crescimento da população, o que sugere que a população está envelhecendo, com os consequentes custos associados à saúde. Além disso, o perfil brasileiro contrasta com dados dos Estados Unidos, Canadá, Europa, Austrália e Nova Zelândia, onde houve uma redução progressiva das fraturas de quadril nos últimos 10 anos. ${ }^{19-21}$

No entanto, mesmo em países onde as fraturas de quadril têm diminuído, há algumas mudanças de tendência. Nos Estados Unidos, após 10 anos de diminuição progressiva na incidência de fraturas de quadril, as taxas de fraturas de quadril em mulheres com 65 anos ou mais pararam de diminuir. Durante esse período, um número menor de densitometrias foi realizado devido à alteração no reembolso e, juntamente com uma menor prescrição de medicamentos para osteoporose, foi interrompida uma redução adicional na incidência de fraturas de quadril. ${ }^{22}$ Pacientes com fratura de quadril devido à fragilidade correm maior risco de fraturas subsequentes. Após uma fratura de quadril, é indicada uma avaliação inicial da densidade mineral óssea da fratura por fragilidade e, caso seja diagnosticada osteoporose, deve-se instalar um tratamento subsequente para evitar futuras fraturas. ${ }^{6}$

Apesar das evidências de que uma ocorrência de fratura de quadril devido à fragilidade é um risco para fraturas secundárias, e os tratamentos disponíveis têm se mostrado extremamente eficientes na redução de fraturas subsequentes, as taxas de tratamento após uma fratura devido à fragilidade variam de 10 a $30 \% .{ }^{6}$ Nosso estudo mostrou que houve um aumento na reabilitação de pacientes com fratura de quadril de 2008 a 2012, atingindo $\sim 40 \%$. No entanto, depois disso, a taxa de reabilitação é estável. O motivo da estagnação das taxas de reabilitação no sistema público de saúde deve ser a saturação dos serviços de reabilitação ou a falta de iniciativa dos médicos para indicar reabilitação para pacientes fraturados ou mesmo a baixa aderência dos pacientes aos procedimentos de reabilitação. Como a reabilitação pode prevenir fraturas secundárias, esse cenário leva à hipótese de que a baixa taxa de reabilitação possa expor um número maior de pacientes em risco de fraturas secundárias, o que, por sua vez, aumenta o número de fraturas de quadril.

Além da reabilitação, os medicamentos antiosteoporose são eficazes na redução das fraturas secundárias causadas pela fragilidade. ${ }^{23}$ No Brasil, alguns medicamentos são oferecidos pelo SUS ou programas especiais patrocinados pelos governos estaduais. Apesar da medicação injetável ter melhor aderência ao tratamento, tanto drogas orais quanto as injetáveis nem sempre são prescritas. Além disso, a baixa adesão ao tratamento em geral pode afetar ainda mais as fraturas secundárias. ${ }^{24}$

O aumento progressivo da incidência de fraturas de quadril no Brasil demonstrado em nosso estudo destaca o impacto nos custos para o SUS, que vem crescendo na mesma proporção e alcançou quase BRL 130 milhões em 2017. Embora o custo total tenha aumentado $40 \%$, esse crescimento se deve exclusivamente a um maior número de internações, uma vez que o custo médio por hospitalização diminuiu $13,6 \%$, mantendo um período de internação semelhante. Além do impacto financeiro, as fraturas de quadril na população idosa resultam em uma taxa de mortalidade $\sim 5,0 \%$ em pacientes com $\geq 50$ anos. Considerando a crescente população de idosos no Brasil, esses dados mostram o impacto na sociedade e a necessidade de ações imediatas para prevenção de fraturas.

Nesse contexto, o ortopedista desempenha um papel central. As fraturas por fragilidade aumentam o risco de fraturas secundárias. Portanto, a intervenção do ortopedista não se resume a realizar cirurgia, consolidação e reabilitação dos pacientes fraturados. É necessário evitar novas fraturas e o tratamento preventivo é crucial. Se o ortopedista não for capaz de realizar mais diagnósticos e tratamentos preventivos, os pacientes devem ser encaminhados para outro profissional para receber o tratamento e o manejo adequados. $\mathrm{O}$ Brasil oferece tratamento para osteoporose por meio de ambulatórios do SUS, também pelo programa Serviço de Ligação de Fratura (FLS, na sigla em inglês).

O FLS é um serviço credenciado pela International Osteoporosis Foundation (IOF) com o objetivo de garantir que todos os pacientes que apresentem fraturas por fragilidade recebam avaliação e tratamento dos riscos de fratura de forma apropriada. Esses serviços são baseados em instituições de saúde secundárias ou primárias em todo o mundo e são considerados a melhor ferramenta para reduzir fraturas em larga escala. O Brasil possui 22 FLS credenciados pelo programa "Capture the Fracture" (Capture a Fratura), ${ }^{25,26} \mathrm{e}$ cerca da metade deles está sediada em hospitais do SUS. Assim, fica claro que o sistema preventivo está disponível para a população brasileira e várias fraturas secundárias poderiam ser evitadas se bem gerenciadas, diminuindo o impacto financeiro e social. Apesar da disponibilidade do FLS, sua implantação e prevenção de fraturas secundárias não fazem parte dos Programas do Ministério da Saúde.

É importante considerar as limitações de nosso estudo. A codificação da CID-10 usada no DATASUS não permite a estratificação entre fraturas por fragilidade e fraturas por impacto. $O$ sistema DATASUS não permite a conexão individual de bancos de dados de hospitalizações (SIH/DATASUS) e ambulatoriais (SIA/DATASUS), portanto, as taxas aqui apresentadas para pacientes reabilitados são estimadas com base no total de cuidados prestados e não por pacientes individuais. Outro ponto a considerar é que avaliamos dados da população coberta pelo SUS que não possui plano de saúde privado. Dados da Agência Nacional de Saúde Suplementar mostram que quase 50 milhões de brasileiros em 2017 eram beneficiários de seguro de saúde privado, o que representa $\sim 25 \%$ da população. ${ }^{27}$ Outro ponto a ser considerado é que os dados obtidos não identificam a origem da fratura; portanto, vários 
casos de fratura de quadril aqui incluídos podem ter origem em traumas e não por fragilidade. No entanto, a epidemiologia das fraturas osteoporóticas confirma nossos achados, pois a maioria das fraturas em idosos ocorre devido à fragilidade. Nossos dados sustentam que a hipótese de que a prevalência de fraturas é maior em homens entre 50 e 60 anos do que entre as mulheres, resultado que provavelmente tem contribuição das fraturas traumáticas. Por outro lado, após os 60 anos, há um aumento nas taxas de fraturas em mulheres, aumentando a prevalência em relação aos homens. Esse fenômeno provavelmente ocorre devido ao efeito da menopausa, que começa por volta dos 45 e 50 anos de idade e, como sabemos, afeta a densidade óssea. ${ }^{28}$ Ao mesmo tempo, as fraturas por trauma são provavelmente mais baixas em homens mais velhos.

No entanto, apesar das limitações de nosso estudo, pudemos demonstrar que na última década há um aumento progressivo das fraturas de quadril devido à fragilidade entre a população $\geq$ 50 anos, estagnação nos tratamentos de reabilitação e redução na média de custo por hospitalização. Outros países com sistema de saúde semelhante ao nosso experimentaram uma diminuição, ou pelo menos uma estabilização anual, na incidência de fraturas de quadril nas últimas 2 décadas. Esse objetivo foi alcançado devido a um melhor gerenciamento dos pacientes, seguido de ações que contribuíram muito para um melhor resultado, como o aumento da conscientização dos cirurgiões ortopédicos e o incentivo a novos FLS. ${ }^{29}$ Em resumo, com base em nossos dados, os cirurgiões ortopédicos precisam estar cientes da importância da reabilitação e do tratamento preventivo após uma fratura de quadril devido à fragilidade, para evitar fraturas secundárias e custos associados.

\section{Suporte Financeiro}

Não houve suporte financeiro de fontes públicas, comerciais, ou sem fins lucrativos.

\section{Conflito de Interesses}

Os autores declaram não haver conflito de interesses.

\section{Agradecimentos}

Os autores agradecem a Tatiana CS Bonetti, Doutora, pela assistência na preparação do manuscrito e redação.

\section{Referências}

1 Abrahamsen B, van Staa T, Ariely R, Olson M, Cooper C. Excess mortality following hip fracture: a systematic epidemiological review. Osteoporos Int 2009;20(10):1633-1650

2 Cummings SR, Melton LJ. Epidemiology and outcomes of osteoporotic fractures. Lancet 2002;359(9319):1761-1767

3 Bliuc D, Nguyen ND, Milch VE, Nguyen TV, Eisman JA, Center JR. Mortality risk associated with low-trauma osteoporotic fracture and subsequent fracture in men and women. JAMA 2009;301(05):513-521

4 Lawrence TM, Wenn R, Boulton CT, Moran CG. Age-specific incidence of first and second fractures of the hip. J Bone Joint Surg Br 2010;92(02):258-261

5 Johnell O, Kanis JA, Odén A, et al. Fracture risk following an osteoporotic fracture. Osteoporos Int 2004;15(03):175-179

6 Stolnicki B, Oliveira LG. Para que a primeira fratura seja a última. Rev Bras Ortop 2016;51(02):121-126

7 Vaile J, Sullivan L, BennettC, Bleasel J. First fracture project: addressing the osteoporosis care gap. Intern Med J 2007;37(10):717-720
8 Hartholt KA, Lee R, Burns ER, van Beeck EF. Mortality from falls among US adults aged 75 years or older, 2000-2016. JAMA 2019; 321(21):2131-2133

9 Beaupre LA, Binder EF, Cameron ID, et al. Maximising functional recovery following hip fracture in frail seniors. Best Pract Res Clin Rheumatol 2013;27(06):771-788

$10 \mathrm{Wu}$ D, Zhu X, Zhang S. Effect of home-based rehabilitation for hip fracture: A meta-analysis of randomized controlled trials. J Rehabil Med 2018;50(06):481-486

11 Pinheiro MdeM, Eis SR. Epidemiology of osteoporotic fractures in Brazil: what we have and what we need. Arq Bras Endocrinol Metabol 2010;54(02):164-170

12 Edelmuth SV, Sorio GN, Sprovieri FA, Gali JC, Peron SF. Comorbidades, intercorrências clínicas e fatores associados à mortalidade em pacientes idosos internados por fratura de quadril. Rev Bras Ortop 2018;53(05):543-551

13 Hungria Neto JS, Dias CR, Almeida JD. Características epidemiológicas e causas da fratura do terço proximal do fêmur em idosos. Rev Bras Ortop 2011;46(06):660-667

14 Loures FB, Chaoubah A, Maciel VS, Paiva EP, Salgado PP, Netto ÁC Cost-effectiveness of surgical treatment for hip fractures among the elderly in Brazil. Rev Bras Ortop 2015;50(01):38-42

15 Soares DS, Mello LM, Silva AS, Martinez EZ, Nunes AA. Fraturas de fêmur em idosos no Brasil: análise espaço-temporal de 2008 a 2012. Cad Saude Publica 2014;30(12):2669-2678

16 Brasil. Ministério da Saúde. Prevenção à osteoporose deve começar na infância, 2011

17 Haentjens P, Autier P, Barette M, Boonen S; Belgian Hip Fracture Study Group. The economic cost of hip fractures among elderly women. A one-year, prospective, observational cohort study with matched-pair analysis. J Bone Joint Surg Am 2001;83(04):493-500

18 IBGE. Projeção da população do Brasil e das Unidades da Federação, 2019

19 Brauer CA, Coca-Perraillon M, Cutler DM, Rosen AB. Incidence and mortality of hip fractures in the United States. JAMA 2009;302 (14):1573-1579

20 Cooper C, Cole ZA, Holroyd CR, et al. IOF CSAWorking Group on Fracture Epidemiology. Secular trends in the incidence of hip and other osteoporotic fractures. Osteoporos Int 2011;22(05):1277-1288

21 Leslie WD, O'Donnell S, Jean S, et al. Osteoporosis Surveillance Expert Working Group. Trends in hip fracture rates in Canada. JAMA 2009;302(08):883-889

22 Lewiecki EM, Wright NC, Curtis JR, et al. Hip fracture trends in the United States, 2002 to 2015. Osteoporos Int 2018;29(03):717-722

23 Cranney A, Tugwell P, Wells G, Guyatt GOsteoporosis Methodology Group and The Osteoporosis Research Advisory Group. Metaanalyses of therapies for postmenopausal osteoporosis. I. Systematic reviews of randomized trials in osteoporosis: introduction and methodology. Endocr Rev 2002;23(04):496-507

24 Kothawala P, Badamgarav E, Ryu S, Miller RM, Halbert RJ. Systematic review and meta-analysis of real-world adherence to drug therapy for osteoporosis. Mayo Clin Proc 2007;82(12):1493-1501

25 International Osteoporosis Foundation (IOF). Capture the Fracture - Map of Best Practice. Disponível em: https://www.capturethefracture.org

26 Marsh D, Åkesson K, Beaton DE, et al. IOF CSA Fracture Working Group. Coordinator-based systems for secondary prevention in fragility fracture patients. Osteoporos Int 2011;22(07):2051-2065

27 Agência Nacional de Saúde Suplementar (ANS). Beneficiários de planos privados de saúde, por cobertura assistencial (Brasil 2009-2019); 2019

28 Viswanathan M, Reddy S, Berkman N, et al. Screening to Prevent Osteoporotic Fractures: Updated Evidence Report and Systematic Review for the US Preventive Services Task Force. JAMA 2018;319 (24):2532-2551

29 Albergaria BH, Chalem M, Clark P, Messina OD, Pereira RMR, Vidal LF. Consensus statement: osteoporosis prevention and treatment in Latin America-current structure and future directions. Arch Osteoporos 2018;13(01):90-90 